\title{
Toward integrated management of cerebral aneurysms
}

\author{
By M. C. Villa-Uriol ${ }^{1,2}$, I. Larrabide ${ }^{2,1}$, J. M. Pozo ${ }^{1,2}$, M. Kim ${ }^{1,2}$, \\ O. Camara ${ }^{1,2}$, M. De Craene $^{2,1}$, C. Zhang ${ }^{1,2}$, A. J. Geers ${ }^{1,2}$, \\ H. Morales ${ }^{1,2}$, H. Bogunović ${ }^{1,2}$, R. Cardenes $^{1,2}$ And A. F. Frangi ${ }^{1,2,3}$ \\ ${ }^{1}$ Centre for Computational Imaging 8 Simulation Technologies in Biomedicine \\ (CISTIB), \\ Information \& Communication Technologies Department, \\ Universitat Pompeu Fabra, c/ Tanger 122-140, E08018, Barcelona, Spain \\ ${ }^{2}$ Networking Research Center on Bioengineering, Biomaterials and Nanomedicine \\ (CIBER-BBN), c/ Tanger 122-140, E08018, Barcelona, Spain \\ ${ }^{3}$ Institució Catalana de Recerca i Estudis Avançats (ICREA), \\ c/ Tanger 122-140, E08018, Barcelona, Spain
}

In the last few years, some of the visionary concepts behind the Virtual Physiological Human (VPH) begun to be demonstrated on various clinical domains showing great promise for improving healthcare management. In the current work, we provide an overview of image- and biomechanics-based techniques that, when put together, provide a patient-specific pipeline for the management of intracranial aneurysms. The derivation and subsequent integration of morphological, morphodynamic, haemodynamic and structural analyses allow to extract patient-specific models and information from which diagnostic and prognostic descriptors can be obtained. Linking such new indices with relevant clinical events should bring new insights on the processes behind aneurysm genesis, growth and rupture. The development of techniques for modelling endovascular devices like stents and coils allows evaluation of alternative treatment scenarios before the intervention takes place and could also contribute to the understanding and improved design of more effective devices. A key element to facilitate the clinical take-up of all these developments is their comprehensive validation. Although a number of previously published results have shown the accuracy and robustness of individual components, further efforts should be directed to demonstrate the diagnostic and prognostic efficacy of these advanced tools through large-scale clinical trials.

\footnotetext{
Keywords: cerebral aneurysms, image segmentation, image processing, morphology, morphodynamics, haemodynamics, computational physiology, structural mechanics, virtual treatment, virtual physiological human
}

\section{Introduction}

Saccular intracranial aneurysms are abnormal focal dilations of cerebral arteries commonly located at the circle of Willis. When they rupture, spontaneous subarachnoid hemorrhage usually follows, causing high morbidity and mortality rates (Brisman et al. 2006). Over the last decade, major improvements have been made in cerebral aneurysm diagnosis and treatment as a consequence on substantial technological breakthroughs on diagnostic and interventional imaging as well as in a 
new generation of therapeutic devices (Wiebers et al. 2003). Despite these advances, still little is known about cerebral aneurysm's genesis, growth and rupture. To better understand these mechanisms from the anatomical and biomechanical points of view, computational models have been developed (Jou et al. 2003; Steinman et al. 2003) and need to be exhaustively evaluated before they become part of the standard clinical practice.

The most common histologic finding in aneurysms is a decrease in the middle muscular layer of the artery, resulting in structural defects that cause localized weakness in the vessel wall (Meng et al. 2007). The combination of these defects with haemodynamic factors, such as wall shear stress, pressure, residence time and flow impingement, are thought to play a significant role in their pathogenesis and thrombosis (Juvela 2003; Kayembe et al. 1984). Indeed, endovascular devices used for aneurysm treatment are designed to modify the local flow conditions. In addition, haemodynamics is bidirectionally related with vascular anatomy: aneurysmal haemodynamics is to a large extent a geometry-driven flow (Cebral et al. 2005) and aneurysm morphology is believed to be the result of a pathology evolution crucially dependent on the local blood flow (Meng et al. 2007), what makes morphological information a likely surrogate of haemodynamics. On the other hand, evidences indicate that aneurysms pulsate over the cardiac cycle and rupture sites are thought to coincide with the areas of larger pulsation (Hayakawa et al. 2005). Also, haemodynamics can be affected by aneurysm and vessels wall compliance (Dempere-Marco et al. 2006).

The methodologies described here have been developed within projects such as @neurIST $\dagger$ and the CDTEAM $\ddagger$ that aim at incarnating into clinical workflows the integration of medical imaging, computational imaging and computational physiology. The vision behind these projects is internationally known as "IUPS Physiome Project" (Hunter et al. 2002; Hunter \& Borg 2003) or, in the European context, as "Virtual Physiological Human (VPH)" (Fenner et al. 2008). The VPH is of great relevance in the current context given its integrative vision of the physiological and computational knowledge. From this perspective, a key challenge in the development of integrative and predictive models for the human physiology is the creation of computational tools that enable the collaborative work between scientists, industry and health care providers to bridge the gap between scientific discovery and clinical applicability.

The goal of this paper is to present a data processing pipeline primarily focusing on the extraction of diagnostic and prognostic indices from medical images and from patient-specific biomechanical models. On the one hand, we are interested in personalized assessment of aneurysm growth and rupture, particularly associating it to image-based computational characterization of intra-aneurysmal flow (Cebral et al. 2005; Dempere-Marco et al. 2006; Radaelli et al. 2007; Geers et al. 2009) as well as to mechanical and morphodynamical features of intracranial aneurysms (Hernandez \& Frangi 2007; Bogunović et al. 2008; Millan et al. 2007; Oubel et al. 2007; Zhang et al. 2009b, a; De Craene et al. 2008, 2009; Balocco et al. 2008). On the other hand, we aim also to develop techniques for the optimization of design and treatment using medical devices like stents and coils used for aneurysm embolization

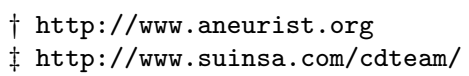


(Radaelli et al. 2008; Larrabide et al. 2008; Kim et al. 2009; Morales et al. 2009; Flore et al. 2009).

\section{Methodology}

At present, three imaging modalities allow to obtain volumetric images of intracranial aneurysms: computed tomography angiography (CTA), magnetic resonance angiography (MRA), and three-dimensional rotational angiography (3DRA). 3DRA provides the highest spatial resolution and in general is used during endovascular treatment. Given the less invasive nature of CTA and MRA, these two modalities are used in standard clinical practice at early diagnostic stages as well as for patient screening and monitoring (Brisman et al. 2006). Time of flight (TOF) MRA is also able to provide some coarse information on the blood flow, and some limited haemodynamic variables such as pressure, could be obtained with intravascular probes. Apart from that, no imaging modality can currently provide detailed description of haemodynamics or wall mechanical properties. However, these can be obtained as the result of computational modelling, potentially involving inverse problem techniques.

Starting from a medical image, the patient's clinical history and a database of medical devices, our data processing pipeline (figure 1) is able to streamline the creation of patient-specific anatomic, structural and haemodynamic models. Those are used to derive robust and reliable quantitative descriptors for patient-specific assessment and treatment planning. Figure 1 provides the overview of the proposed pipeline and the methodologies involved in the creation of such models. The first and probably most critical step in personalized vascular modelling, on which almost all other steps are dependent, is obtaining a proper anatomical representation from the medical images using image segmentation techniques. Complete 3D morphological descriptors can be obtained directly from the vascular models, susceptible to be linked with aneurysm growth or rupture. Recovering the aneurysm wall morphodynamics can be relevant on its own for the clinical evaluation. In addition, this information is essential in order to estimate the structural properties of the aneurysm wall. Computational models offer the possibility of integrating the limited information available from medical images and estimating these nonobservable parameters using data assimilation techniques. Computational Haemodynamics (CHD) simulations (Cebral et al. 2005) are able to provide a description of patient-specific haemodynamic variables with high spatial and temporal detail. Computational models have been developed to represent the virtual implantation of endovascular devices (stents and coils).

\section{(a) Anatomical modelling and morphological analysis}

\section{(i) From medical images to anatomical models}

The present processing pipeline relies on the availability of accurate patientspecific vascular models extracted from medical images. In general, for image-based pipelines, the process used to extract such models needs to be robust to ensure accuracy and reliability. This task is difficult and challenging, being heavily influenced by the underlying image quality and the complexity of the vascular geometry. Our knowledge-based algorithm (Hernandez \& Frangi 2007) based on Geodesic Active 


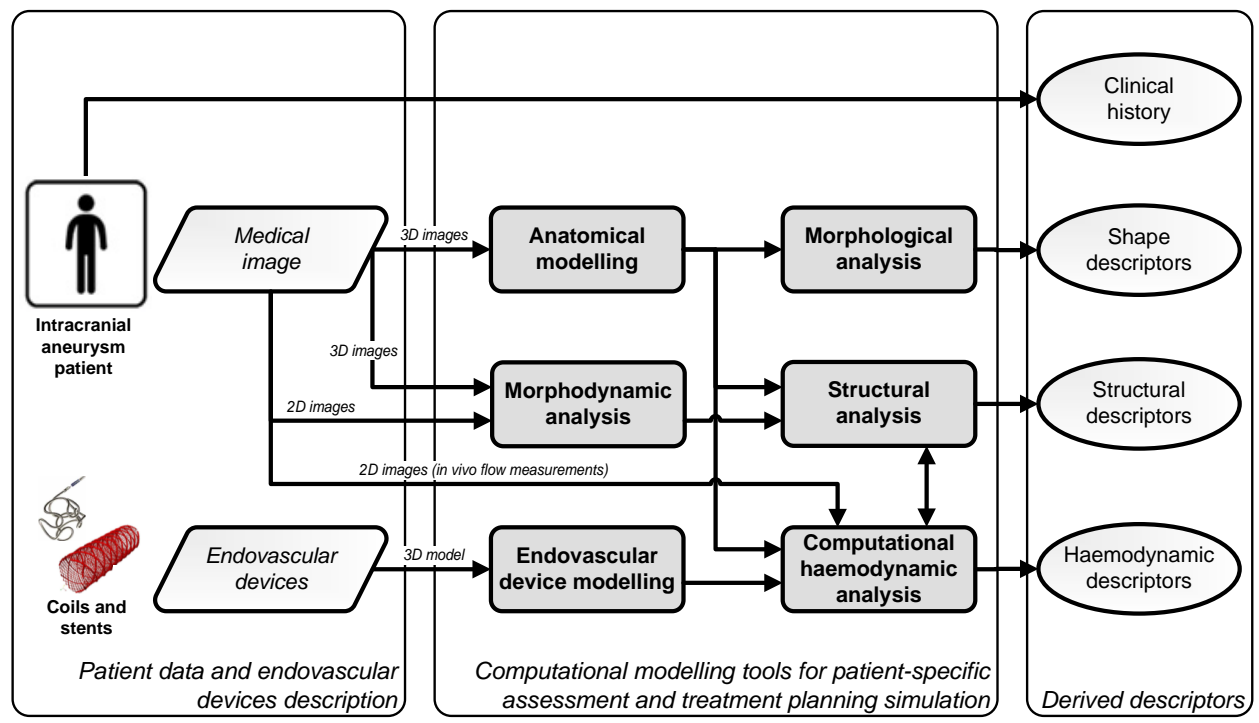

Figure 1. Data processing pipeline for patient-specific assessment and treatment planning.

Regions (GAR) was devised for the automatic segmentation of the entire vascular tree. This approach contrasts with other more interactive approaches focused on localized vascular segments (Castro et al. 2006; Antiga et al. 2008; Chang et al. 2009). GAR consisted in a geometric deformable model with an associated energy functional that incorporated second-order multiscale features together with image gradient magnitude. Bogunović et al. (2008) presented an image intensity standardization (IIS) method aimed at the standardization of intensity ranges of tissue classes in routine medical images and showed its applicability to the vascular segmentation of 3DRA images. The combined use of IIS and GAR has been extensively used in our group and validated with cerebrovascular 3DRA, CTA and MRA images (Hernandez \& Frangi 2007; Radaelli et al. 2007; Bogunović et al. 2008; Radaelli et al. 2008; Geers et al. 2009; Kim et al. 2009). For example, in the case of 3DRA and MRA images, the extracted vascular models have been recently compared and validated against a gold standard built from manual measurements performed by clinicians. The preliminary results indicate that average absolute differences for 3DRA and MRA with respect to the gold standard were $0.2 \mathrm{~mm}$ and $0.3 \mathrm{~mm}$ respectively, values below the corresponding image resolutions. Moreover, GAR and IIS methods are generic and could be used to segment other tissues and/or from other imaging modalities by providing the appropriate training set to the method. This is the case in a recent work (Krissian et al. 2008; Schaap et al. 2009) in which they were part of a method to extract coronary artery centrelines in a minimally interactive fashion from CTA images.

\section{(ii) From anatomical models to aneurysm morphological analysis}

Interventional neuroradiologists and neurosurgeons are used to decide treatment based on morphological and positional factors such as aneurysm size, appearance 
and location. Based on these observations, several works (Rohde et al. 2005; Ujiie et al. 1999; Ma et al. 2004; Raghavan et al. 2005) have linked basic geometrical aneurysm features to rupture risk, e.g. aspect ratio.

Contrary to the widespread clinical practice and most proposed indices, we contend that aneurysmal morphology has to be characterized considering fully its tridimensionality as opposed to the use of a few linear dimensions extracted from projectional imaging. For this reason, in Millan et al. (2007) an efficient and novel methodology for 3D shape characterization of intracranial aneurysms based on moment invariants was proposed. Two different moment types were considered: Geometrical Moment Invariants (GMI) (Lo \& Don 1989) and 3D Zernike Moment Invariants (ZMI) (Novotni \& Klein 2004). In particular Zernike moments provide a set of 3D morphological descriptors that are complete, meaning that, up to a level of detail, the shape can be recovered from their moments. In contrast with previously proposed descriptors, aneurysms were isolated including part of their parent vessels, whose configuration, in relation to the aneurysm, should play an important role in the resulting blood flow. GMI and ZMI were computed for a database containing 53 patients with a total of 31 ruptured aneurysms and 24 unruptured aneurysms. As a result (Millan et al. 2007), ZMI indices proved to be more robust with respect to GMI, and provided a more reliable discrimination between ruptured and unruptured aneurysms. Using these descriptors, correct rupture prediction rates of $\simeq 80 \%$ were achieved in contrast to $66 \%$ found when the aspect ratio index was considered.

Apart from their completeness, 3D Zernike moments are orthonormal, which provides the advantage of their Euclidean distance being a natural and geometrically meaningful distance measure. Computing this distance in the space of ZMIs has the additional advantage that the pose dependency is excluded. Hence, the computation of these distances could provide a mean to efficiently compare the morphological characterization of an aneurysm from a new patient to other previously treated aneurysms with their morphological descriptors already stored in a database. The potential integration of this functionality into a more general clinical decision support system could provide a list of similar aneurysms. The combination of morphology with haemodynamic and biological factors could help to estimate the rupture risk and choose accordingly the best treatment option at the time.

\section{(iii) Quantification of volumetric changes}

The quantification of size and shape changes in aneurysm geometry over time is a critical issue for quantifying aneurysm stability. More specifically, the stability of aneurysms after endovascular treatment has been an issue under debate for many years, and high rates of aneurysm recurrence after coiling have been reported (up to one third of treated aneurysms as reported in Raymond et al. (2003)). In this perspective the accurate detection and quantification of local shape changes in the aneurysm is critical for monitoring aneurysm stability and decide for (re)treating.

Our group investigated the use of automatic non-rigid image registration methods to produce local deformation maps as quantified by the Jacobian of a non-rigid transformation (De Craene et al. 2008). State of the art intensity-based non-rigid registration algorithms were applied to produce local volume change maps between sequential images obtained at follow-ups of endovascular aneurysm coiling. This not 
only permits reconstructing volume temporal evolution of coil and aneurysm, but also computating local growth maps showing the relative lengthening of a point of the aneurysm wall rather than its absolute displacement as performed by Boussel et al. (2008). In De Craene et al. (2009), the methodology was extended to account for very large deformations in the case of severe aneurysm growth. This was performed by a novel approach using diffeomorphic non-rigid registration.

\section{(b) Morphodynamic and structural analyses}

To circumvent the well-known difficulties of the in vivo measurements of wall thickness and wall mechanical properties (Taylor \& Humphrey 2009), two methodologies are presented below. First, wall motion estimation techniques from 2D and 3D images, and second a data assimilation framework that based on wall motion measurements plus boundary conditions (pressure and inflow velocity waveforms) is able to estimate aneurysmal wall tissue characteristics.

\section{(i) From medical images to morphodynamic analysis}

In Dempere-Marco et al. (2006) and Oubel et al. (2007) our group proposed a motion estimation method from dynamic digital subtraction angiography (DSA) using 2D non-rigid image registration. Since DSA images were captured from a single point of view, motion was only partially recovered. Recently, we presented in Zhang et al. (2009b) a technique to model patient-specific pulsatility of intracranial aneurysms over one cardiac cycle, using a single 3DRA acquisition. Aneurysm morphology at a given time instant was estimated from its temporal vicinity by matching projections of a deformed 3D reference volume to the corresponding reduced set of 2D projections in a weighted scheme. In Zhang et al. $(2009 a)$, this technique was extended to a $4 \mathrm{D}$ scheme by simultaneously matching the forward projections of a sequence of the temporally deformed 3D reference volume to the entire 2D measured projection sequence. In addition, two strategies were introduced to reduce the high memory and computational costs required for processing large 3D and 2D datasets. An advantage of this methodology for its clinical take-up is that it only requires one of the standard acquisitions performed during an endovascular treatment, eliminating the need of exposing the patient to additional radiation dose.

\section{(ii) From anatomical models to the estimation of aneurysm mechanical properties}

A data assimilation framework can be designed to estimate the mechanical parameters of a computational model of the cerebral aneurysm wall based on available wall pulsation measurements as Kroon \& Holzapfel (2008) applied on idealized (spherical, axisymmetric) aneurysm geometries. In Balocco et al. (2008), we incorporated information provided by in vivo imaging data into a data assimilation framework for estimating regional mechanical properties of cerebral aneurysms. The deformation field capturing aneurysm wall pulsation was obtained applying a registration technique to a pair of simulated MR images. In order to better distinguish between different wall pulsation patterns, strain maps were computed from the deformation field. A region clustering technique was then applied to these strain maps in order to speed up the estimation of the mechanical parameters. The parametric 
biomechanical model generated the modelled aneurysm morphology in systole from: the diastolic morphology segmented from subject-specific images; a given regional distribution of the elasticity parameters; and a set of boundary conditions. The structural mechanics behaviour of the model was controlled by regional elasticity parameters, which were initialized with values based on ex vivo experiments available in the literature. Subsequently, these parameters were optimized at the inverse problem stage to minimize the difference between two aneurysm morphologies in systole: the one given by the biomechanical model; and the one generated from applying the deformation field capturing the aneurysm wall pulsation to the diastolic morphology. A local pressure distribution that was estimated with computational fluid-dynamics was introduced into the structural mechanics as an additional constraint.

\section{(c) Computational haemodynamic analysis and endovascular device modelling}

The ultimate goal of cerebral aneurysm treatment is to permanently exclude the aneurysm from the rest of vascular circulatory system and to return the blood flow to normal physiological conditions. Among the multiple techniques pursuing this objective, coiling (Guglielmi et al. 1991) and stenting (Brisman et al. 2005; Lylyk et al. 2005) have become the preferred options over the traditional surgical clipping. The use of stents is rapidly evolving from being merely a support device for the coil to becoming an active flow diverting device (Vanninen et al. 2003).

Currently, CHD is used to investigate the role of haemodynamics in the initiation, growth and rupture of patient-specific vascular models. Moreover, the flow and device modelling techniques enable the assessment of the pre- and post- treatment (coiling, stenting) haemodynamic conditions in the aneurysms.

In the proposed processing pipeline, we introduce a number of techniques able to simulate these alternative scenarios combining image- and biomechanics-based patient-specific information and, where available, with device information.

\section{(i) Computational haemodynamic analysis}

CHD requires proper patient-specific boundary conditions in terms of flow rate or pressure to solve the governing equations. These flow boundary conditions can be obtained from phase-contrast magnetic resonance images for the main branches of the circle of Willis. Nevertheless, since patient-specific measurements are seldom available, reference data obtained from normal volunteers (Ford et al. 2005a; Alastruey et al. 2007) or 1D/0D lumped parameter models (Blanco et al. 2007; Olufsen et al. 2002) are often employed instead. To model the blood flow in cerebral aneurysms the assumptions of incompressible, laminar flow and Newtonian/nonNewtonian fluid in the rigid/compliant vessel walls are commonly used and accepted within the community (Cebral et al. 2005).

In order to introduce these techniques into routine clinical practice, validation against ground truth in vivo flow measurements is mandatory. Due to the fact that such data can not possibly be obtained with the technology available nowadays, different alternatives have been proposed. A number of authors have compared simulation results with experimental flow measurements in idealized phantoms (Hoi et al. 2006) and anatomically realistic replicas (Ford et al. 2008). Another alternative 
corresponds to the comparison of simulation results with gross haemodynamical features obtained with routine image modalities.

In particular, Cebral et al. (2004) compared the isovelocity surfaces extracted from the haemodynamic simulation to isointensity surfaces in TOF-MRA images obtained by Satoh et al. (2004). They found a good agreement in the aneurysmal inflow region.

In two separate works, Ford et al. (2005b) and Calamante et al. (2003) presented a strategy to simulate the transport of the contrast agent in cerebral vasculature and produced visualizations which reproduce conventional angiography. This technique, named virtual angiography, allows a direct qualitative comparison of simulation results with high frame rate angiographic images routinely acquired during treatment. We believe that the use of such visualization techniques (Ford et al. 2005b; Cebral et al. 2007) will play an important role in the clinical acceptance of CHD in the clinical practice.

\section{(ii) Virtual stenting}

Previous works have modelled, in a simplified manner, the presence of endovascular stents in cerebral vessels with aneurysms (Cebral \& Löhner 2005). Larrabide et al. (2008) proposed a Fast Virtual Stenting (FVS) methodology based on constrained deformable simplex models, where a second order differential equation was used to deform a simplex mesh under the effect of physical constraints such as stent mesh shape, size, and stent radius. This work extended the method proposed by Delingette (1999). These parameters were selected because they were relatively easy to obtain from the stent companies and were also sufficient to describe the global stent geometry without the need of performing detailed structural simulations As a result, the technique effectively embeds the geometrical properties of the stent described above and achieves favorable execution times of the order of one minute.

In a recent study, Flore et al. (2009) compared structures of stents deformed by two different methodologies, FVS and Finite Element Analysis (FEA). A series of parametric vascular models were designed varying stent geometry, vessel and aneurysm neck sizes, using for the release both methods. Results show good agreement between the two methodologies accompanied by a significant reduction (1000:1) in the computational cost of FVS.

\section{(iii) Virtual coiling}

Simulation of endovascular coils is challenging because of their geometrical complexity. Their final placement depends on the coil design and on the morphology of the aneurysm. They also depend on the number of coils inserted, and the exact order and operator's skills and aims when they are inserted. To simulate the haemodynamics of coiled aneurysms, a simplified model using porous media has been proposed (Liang-Der et al. 2004; Kakalis et al. 2008). In this model, the distribution of coils in an aneurysm have been considered to be homogeneous and isotropic. Alternatively, the coils have been modelled with overlapping spheres along the axis of curved filaments (Cebral \& Löhner 2005) or straight cylinders (Narracott et al. 2005). However, none of them were able to model the coil embolization with realistic high packing rate of an aneurysm.

Article submitted to Royal Society 
Morales et al. (2009) proposed a methodology to simulate the coiling based on medical images and treatment data. In this algorithm, the coil tip advances while minimizing the influence of potential energy associated to the vessel wall boundaries and coil themselves. The energy used by the algorithm to advance the coil is the combination of four terms: the energy field from the vessel wall, the coil surface, the coil physical constraints, and the coil retreat which allows the coil to retreat and advance in a different direction when a dead-end is found. This algorithm allowed to achieve the clinically reported maximum coil packing rates $(\sim 40 \%$ in Sluzewski et al. $(2004))$ in realistic aneurysm geometries. Moreover, it opens the way for further studies concerning pre- and post-treatment outcomes in patients' with diverse arterial morphologies (aneurysm shape, size, and parent vessel structure, etc.).

\section{Results}

The reliability of the proposed pipeline needs to be assessed by testing the reproducibility and the sensitivity of its individual components. Multiple aspects need to be evaluated and we provide several examples illustrating the accuracy of the resulting model in predicting the physical reality and the reproducibility of the modelling process.

First, we evaluate the impact of the image modality in patient-specific computational haemodynamics studies. The results justify the use of CHD for understanding vascular disease progression. Second, we present two methodologies that combined could provide an estimation of cerebral aneurysm wall mechanical properties based on pressure and inflow velocity waveforms, as well as in wall motion estimated from images. Finally, we describe some of the efforts done towards the validation and assessment of the techniques used to simulate virtual treatments (stenting and coiling) as well as a technique to quantify the observed volume changes in patients presenting coil compaction and aneurysm growth.

\section{(a) Patient-specific computational haemodynamics}

(i) Reproducibility of patient-specific computational haemodynamics between $3 D R A$ and $C T A$

One major strength of the proposed modelling pipeline is its applicability to the variety of image modalities used throughout the patient care cycle. In a recent study, Geers et al. (2009) studied the differences between vascular models derived from 3DRA and CTA and the subsequent differences in the haemodynamic simulations.

Four aneurysms on the middle cerebral artery M1-M2 bifurcation were imaged within a one-day time interval with both 3DRA and CTA. For both image modalities a haemodynamic model of each aneurysm was created. The lower contrast and spatial resolution of CTA gave rise to differences in the vascular models. On the one hand, the segmentation algorithm had more difficulties to separate vascular structures that were close to each other. As a result, the aneurysm neck appeared to be wider, which altered the flow rate into the aneurysm as well as other haemodynamic variables. On the other hand, contrast values of small vessels of less than $1 \mathrm{~mm}$ in diameter were more blurred and the segmentation algorithm failed to recognize them. This gave often rise to a higher flow rate in the parent vessel and, 


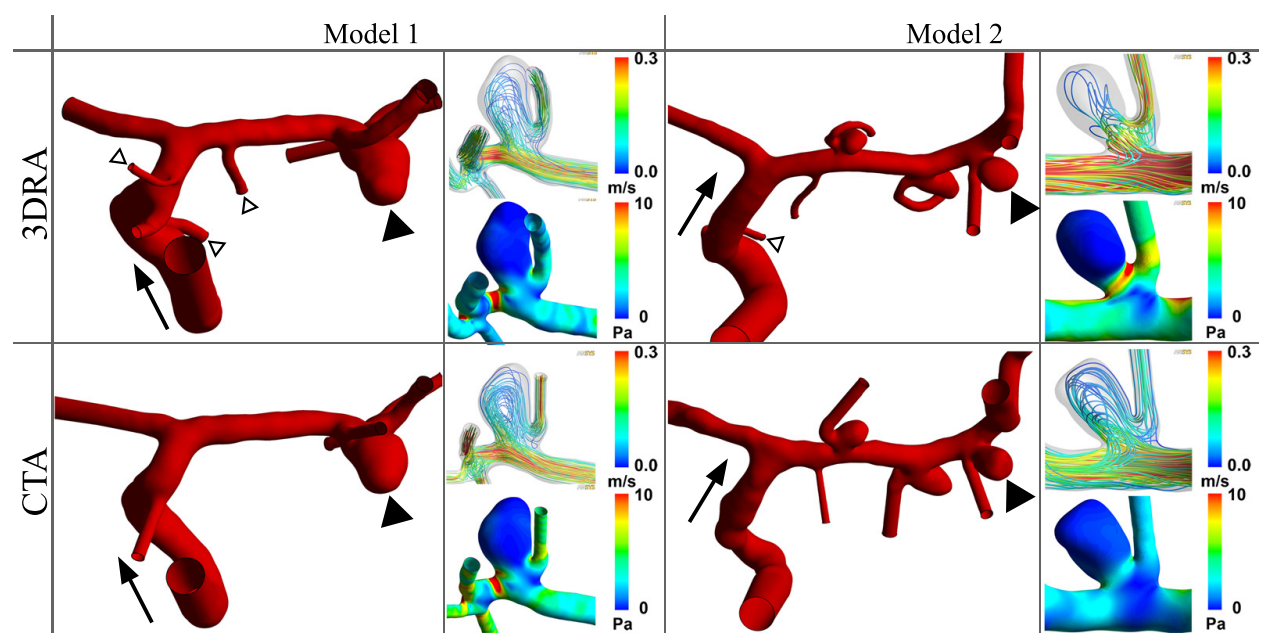

Figure 2. Two patient-specific vascular models extracted from 3DRA and CTA. Flow direction (arrow) and aneurysm (black triangle) are indicated. Small vessels that were not successfully reconstructed in the CTA model are indicated in the 3DRA model (white triangles). Streamlines color-coded by the velocity magnitude (top) and a color map of the wall shear magnitude (down) visualize the time-averaged flow field.

therefore, in the aneurysm. Overall, quantitative haemodynamic measurements did give substantially different results (in some cases up to a $40 \%$ ), but qualitatively the main flow characteristics were found to be well-reproduced between the 3DRAand CTA-based simulations.

\section{(ii) Understanding aneurysm rupture: Mirror aneurysms}

In Radaelli et al. (2007) eight cases of mirror cerebral aneurysms were analyzed through the combination of clinical observations and CHD models. Mirror cerebral aneurysms refer to two aneurysms in the same patient, symmetrically located in the cerebral vasculature with respect to the mid-sagittal plane.

Mirror aneurysms enable to isolate factors like aneurysm morphology and haemodynamics from others factors such as segmental localization, systemic factors, genetics or other patient-specific clinical history data to understand rupture mechanisms. In this case, the patient sample was composed of seven women and one man, between 32 and 76 years of age. In seven cases rupture occurred in one side, while in one case both aneurysms were unruptured. Aneurysm size and just aspect ratio did not differ significantly between the ruptured and the unruptured groups in these samples. However, asymmetry between the two sides of both, the circle of Willis and intracranial segments of the internal carotid artery (ICA), was notable. The vascular structure including size, length, angle, and curvature strongly affected aneurysm haemodynamics and as a result these had an impact on the aneurysms rupture status. 


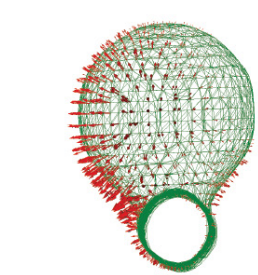

(a)

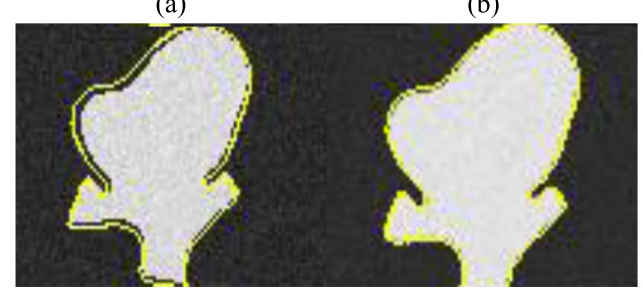

(e)

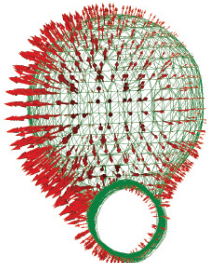

(b)

(f)

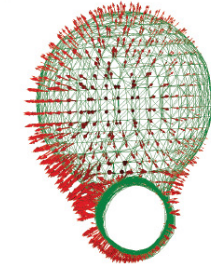

(c)

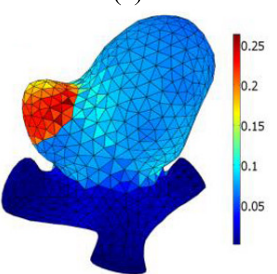

(g)

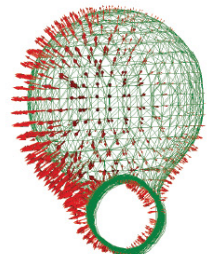

(d)

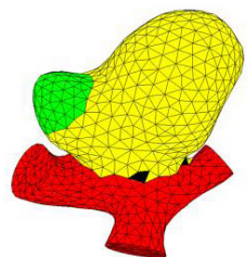

(h)

Figure 3. Four time-points (a-d) of reconstructed wall pulsation from silicone phantom experiments. Simulated images of a diastolic (light gray area) and systolic (yellow contour) aneurysm geometry before (e) and after (f) applying a registration algorithm to recover wall pulsation. Radial strain maps $(\mathrm{g})$ of the recovered wall pulsation. Clustering results (h) distinguishing three regions (bleb, dome, vessel) with different mechanical properties.

\section{(b) Towards the estimation of cerebral aneurysms wall mechanical properties}

\section{(i) Feasibility of estimating cerebral aneurysm wall motion from 3DRA}

We have evaluated the performance of the morphodynamic methodologies presented in (Zhang et al. 2009b,a), using digital and physical pulsating aneurysm phantom models. Both types of phantom data were designed to represent aneurysms with pulsation ranges consistent with literature values. The imaging conditions matched what 3DRA imaging suites used in standard clinical practice offer nowadays. Experiments with digital phantoms were performed under controlled conditions, allowing to quantitatively evaluate the sensitivity of our method to various factors. Results showed estimation errors below $10 \%$ of the maximum pulsation, which in general presented subvoxel wall displacements. Experiments simulating disturbances such as head movements, inhomogeneous contrast agent mixing, scattering and quantum noise, etc., slightly increased the estimation error, which still remained below $20 \%$ in most cases. Physical phantom experiments allowed to demonstrate the feasibility of pulsation estimation under clinical conditions. The simulated projections from the estimated volume images matched the corresponding original ones, which was not the case from those of the reference volume (figure 3). In terms of reducing the high memory and computation costs for processing large 3D and $2 \mathrm{D}$ datasets, a factor about 30-40 times was achieved from the tested data while preserving the accuracy of the motion estimation.

\section{(ii) Feasibility of estimating regional mechanical properties of cerebral aneurysms}

We designed several in silico experiments to test the robustness of the data assimilation framework developed for estimating regional mechanical properties of cerebral aneurysms. The in silico experiments could be classified into two categories: 
the ones studying different aspects of the aneurysm geometries used in our analysis; and the ones investigating the influence of the wall motion accuracy on final results.

For the in silico experiments centered in the geometry, the proposed workflow proved to be very robust with respect to different input geometries, with percentages of true positives above $98.2 \%$ for all different geometries. Increasing the number of blebs in the aneurysm moderately decreased the performance. On the other hand, the choice of the registration configuration had a substantial effect on the recovered stiffness distribution and estimated values. Different spatial resolutions using simulated MR images resulted in differences in the workflow performance.

According to our in silico experiments, $0.1 \mathrm{~mm}$ was the minimum image resolution required for the proposed data assimilation framework to distinguish aneurysm regions with different mechanical properties. These findings were in agreement with results obtained by Zhang et al. (2009b) where we concluded that cerebral aneurysm wall pulsation could be recovered from registration techniques applied on volumes reconstructed from projections having a spatial resolution of $0.155 \mathrm{~mm}$ per dimension. Unfortunately, a resolution of $0.1 \mathrm{~mm}$ is not easily achievable in clinical routine with standard MR acquisition protocols. However, several research groups are actively developing acquisition protocols and imaging systems to improve the spatial resolution in neurovascular images (Rudin et al. 2008; Ganguly et al. 2003).

(c) Intracranial aneurysm treatment planning and assessment: applications and use of virtual stenting

(i) Reproducibility of haemodynamical simulations in a subject-specific aneurysm model with endovascular stent

Extensive analyses of aneurysmal haemodynamical changes induced by stent deployment have been provided by both in vitro and CHD studies (Cebral \& Löhner 2005). These observations suggest the importance of considering patient-specific anatomy to assess the performance of a specific stent design and study its relation with clinical events and post-implant complications. Despite the growing interest in this topic, it is currently difficult to assess the level of technological advancement and the reproducibility of image-based haemodynamical simulation techniques when applied to stented patient-specific models.

The Virtual Intracranial Stenting Challenge (VISC) international initiative sought fulfilling this aim by promoting a multicentre-controlled benchmark to analyze differences induced by diverse grid generation and CHD technologies. The challenge also provides an opportunity to survey available technologies currently adopted by international teams from both academic and industrial institutions for constructing computational models of stented aneurysms. The results of the 2007 edition of the challenge were reported in Radaelli et al. (2008) and demonstrated the ability of current strategies in consistently quantifying the performance of three commercial intracranial stents, and contributed to reinforce the confidence in haemodynamic simulations, thus taking a step forward towards the introduction of simulation tools to support diagnostics and interventional planning.

Article submitted to Royal Society 


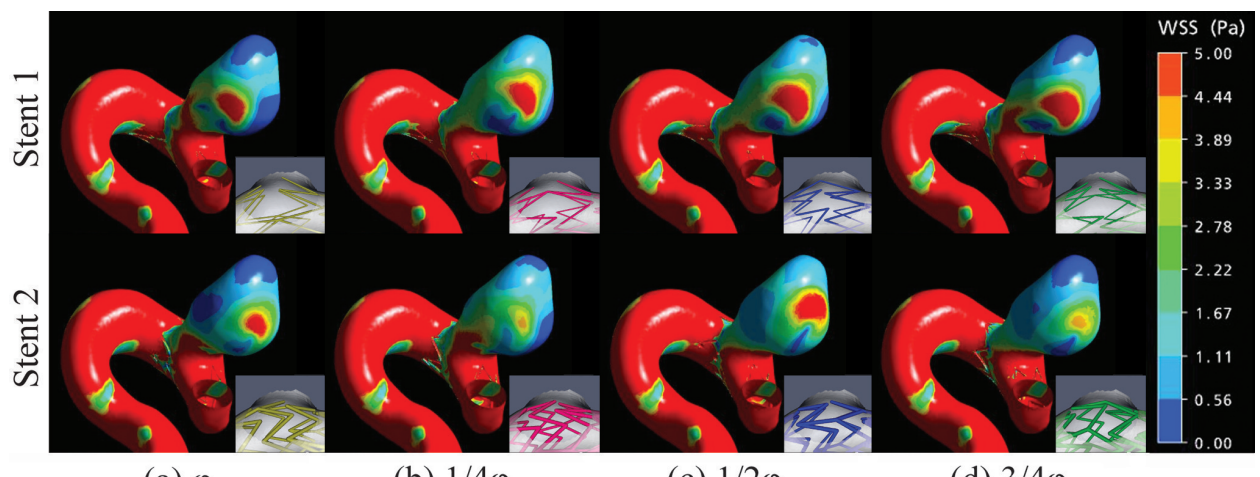

(a) $\varphi$

(b) $1 / 4 \varphi$

(c) $1 / 2 \varphi$

(d) $3 / 4 \varphi$

Figure 4. Influence of stent orientation in WSS for Neuroform and Zilver. Stent orientation is indicated as a fraction of $\phi$, which represents the angular axial symmetry of the stent $\left(180^{\circ}\right.$ degrees). A detailed view of the deployed stent in the aneurysm neck region is shown at the bottom right corner of each WSS plot. Note that for Zilver, the variations of WSS with respect to the stent orientation are evident.

\section{(ii) Influence of stent axial orientation on patient-specific haemodynamics}

Several computational haemodynamic studies have been carried out to clarify the effect of stents on aneurysmal flow (Stuhne \& Steinman 2004; Cebral \& Löhner 2005; Liou \& Li 2008; Kim et al. 2008). These studies concluded that the flow in a stented aneurysm is influenced by multiple factors of the stent geometry such as strut size, and stent porosity.

To study the impact of the stent positioning, Kim et al. (2009) investigated the influence of stenting with various axial orientations on a saccular aneurysm haemodynamics. Two commercial stents were modeled in this study: a Neuroform stent (Boston Scientific, Natick, MA) and a Zilver stent (Cook medical Inc., Bloomington, IN). Both stents were virtually released using the FVS algorithm (Larrabide et al. 2008) in four different axial orientations to fit into the luminal surface of a patient-specific internal carotid artery model with a lateral aneurysm. Computational haemodynamic analyses were carried out for the unstented and stented aneurysm models (figure 4). The intra-aneurysmal flow for stented models show disturbed and complex flow patterns while the flow activity and the forces acting on the aneurysm wall are generally alleviated. The influence of the axial orientation of the stent on the aneurysm haemodynamics is more significant for Zilver which has a larger strut size compared to Neuroform. Interestingly, the flow activity in the aneurysm is rather increased when the intra-luminal scaffolding of stent is not sufficient.

The combined use of CHD with virtual stenting techniques provides a feasible mean to investigate the stent induced haemodynamic alterations in patient-specific aneurysm models, not only facilitating the personalized selection of an optimal stent prior to intervention, but also providing tools to medical device companies for exploring new designs. 
(d) Intracranial aneurysm treatment planning and assessment: applications and use of virtual coiling

(i) Quantification of coil compaction and aneurysm growth

After coiling, aneurysm recurrence can be caused by the compaction of the coil inside the aneurysm, by the growth of the aneurysm, which makes the coil migrating depending on thrombosis and flow-induced forces inside the aneurysm, or the co-occurrence of the both phenomena.

In clinical practice, the neuroradiologist usually detects and quantifies aneurysm recurrence by performing manual measurements and visual inspection of images acquired at follow-up. Coil compaction and aneurysm growth are generally assessed by looking at the presence of a residual neck, which can be observed after subtracted reconstructions in 3DRA, DSA, or MRA.

Although the presence of a residual neck clearly assesses the reperfusion of the aneurysm, it does not allow to determine if it is due to coil compaction or aneurysm growth. In De Craene et al. (2008), an image-processing pipeline was applied to series of 3DRA images obtained in four patients at different controls separated from two months to two years. The evolution of coil and aneurysm volumes along the period was obtained separately, which allows distinguishing between coil compaction and aneurysm growth. On the four cases studied, aneurysm recurrence was always associated to aneurysm growth, as opposed to strict coil compaction. Figure $5(\mathrm{a}-\mathrm{c})$ shows the results for one of these cases.

\section{(ii) Influence of coil packing rate and configuration on intracranial aneurysm haemodynamics}

In Morales et al. (2009), we introduced a virtual coiling algorithm and investigated the influence of the coil packing rate and coil configuration (distribution and structure of deployed coils) on intra-aneurysmal haemodynamics. Here, one internal carotid aneurysm was virtually coiled with four different packing rates $(13 \%, 20 \%$, $25 \%$, and $32 \%$ ), each one of them with three different coil configurations (labelled as A, B and C). CHD analyses were performed both in the untreated and treated aneurysm models (figure $5(\mathrm{~d})$ ). The results reveal that aneurysmal flow velocity and WSS were reduced by coiling even for low packing rates. Also, we observed that WSS near the aneurysm ostium drastically increased due to interference introduced by the coils. Finally, the authors found that the haemodynamic differences due to coil configurations were negligible when the coil packing rate was high (above 20\%) which was observed in a clinical database (Piotin et al. 2000). Thus, it has been concluded that the virtual coiling technique has the potential to become a valid tool to investigate the post-treatment aneurysm haemodynamics.

\section{Discussion}

An image-based computational modelling data processing pipeline has been proposed to achieve an advanced management of cerebral aneurysms. As a result, personalized vascular models are created and used to derive a collection of descriptors. When assembled together, such descriptors could possibly help finding new associations with clinical events such as the aneurysm genesis, growth, rupture or 


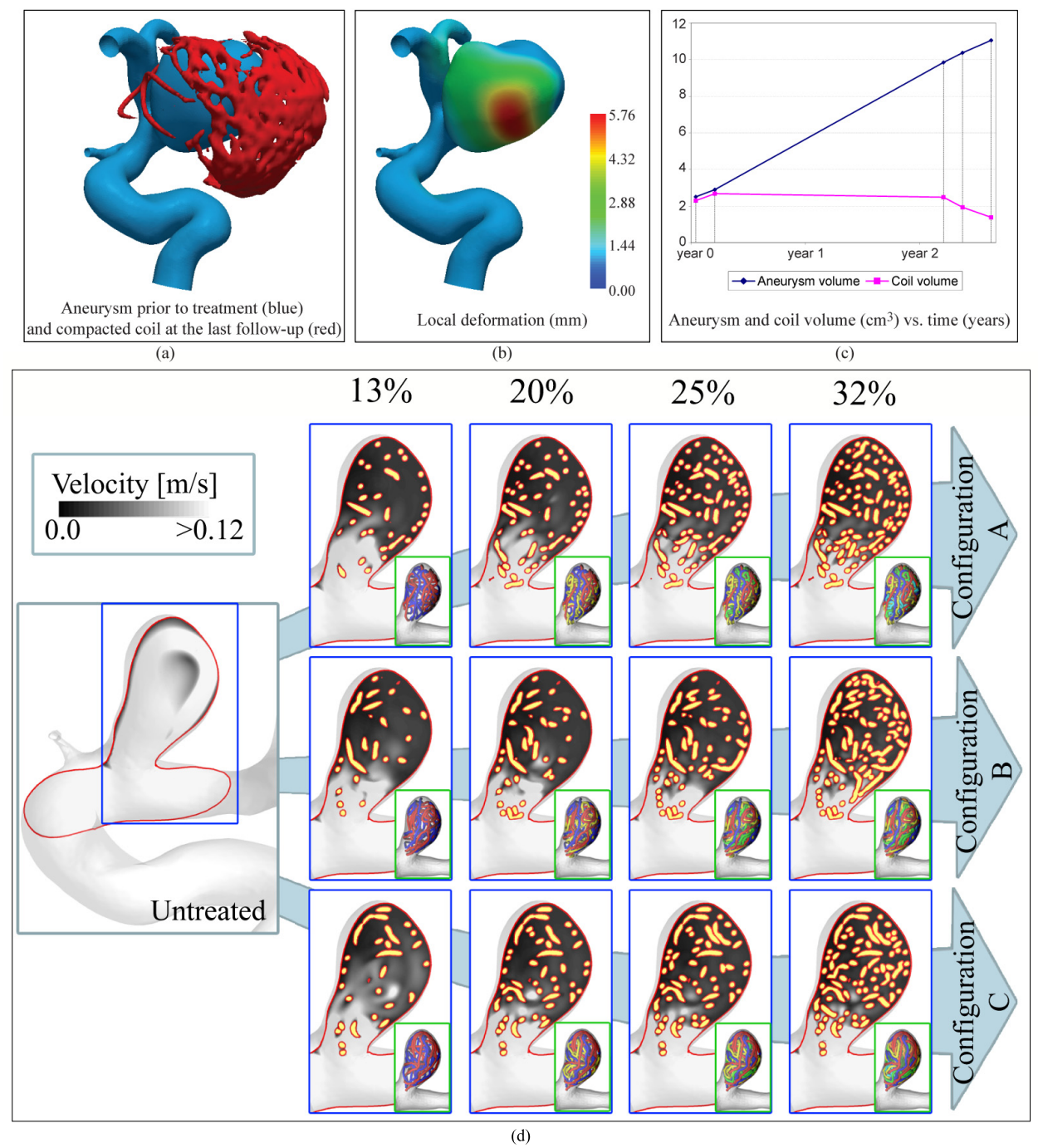

Figure 5. (a-c) Example of quantification of aneurysm growth and coil compaction. (a) Blended view of the aneurysm surface prior to the first treatment and the coil geometry at the last follow-up. (b) Local growth map in the aneurysm between the first and last treatment. (c) Coil and aneurysm volume evolution vs. time curves. (d) Virtual deployment of the coils with different configurations (A, B and C) and packing rates $(13 \%, 20 \%, 25 \%$ and $32 \%$ ). The deployed coils are shown in different colours. Gray level indicates peak systolic velocity contour, on a middle cut plane in the untreated and treated aneurysm models.

post-treatment reopening. Once these have been established, they could provide more comprehensive information to clinicians in assessing the situation of a particular patient. The off-line evaluation of alternative treatment scenarios in presence of different endovascular devices should also be possible comparing the aneurysm haemodynamic conditions before and after treatment. And from the device man- 
ufacturer point of view, such techniques could be used to explore new designs or improve existing ones.

One remarkable feature of this approach is that it relies on an automatic and reproducible image segmentation technique (Hernandez \& Frangi 2007; Bogunović et al. 2008) increasing the reproducibility and consistency of the subsequent analyses. Another important characteristic is that it offers a plausible option for the indirect in vivo estimation of aneurysm wall mechanical properties. Wall motion displacements (Oubel et al. 2007; Zhang et al. 2009b,a) together with flow and pressure measurements are used to optimize these parameters using a data assimilation framework (Balocco et al. 2008). Unfortunately, the standard imaging conditions currently used in clinical practice still pose difficulties in terms of spatial resolution, but they are likely to be overcome in the near future. The use of computational models for the early assessment of aneurysm endovascular treatment can provide a meaningful way to select an optimal treatment for a specific patient. Such techniques allow to reproduce different treatment situations like the use of different stents (Larrabide et al. 2008), variability in the stent position (Kim et al. 2009), and different coil packing rates (Morales et al. 2009), among others. As demonstrated in the VISC 07 Challenge (Radaelli et al. 2008), such studies are reproducible, even when developed by distributed teams.

Although it was outside the scope of this paper to provide a comprehensive validation of the presented pipeline, we have presented for its various components several validation studies that focused on assessing the accuracy, the reproducibility and the repeatability of the results where appropriate. Some of the components were also evaluated as part of the patient-specific image-based simulation pipeline developed under the umbrella of the @neurIST project (Singh et al. 2009). These and other similar studies have been able to build enough evidence on the accuracy and reproducibility of the results of the morphological and haemodynamic analyses and have hinted towards their potential value as diagnostic and prognostic indices. However, to fully ascertain these conclusions and have an impact on the standard-ofcare in clinical practice, further multi-centric trials will need to be performed where the application of these tools yields indices which have demonstrated sufficient predictive power to be introduced in clinical routine.

Cerebral aneurysms are pathological dilations of the vascular wall that are localized in space and result from complex processes evolving in time. Such aneurysms are integrated within the cerebral circulation within the circle of Willis, which itself is integrated within the whole circulatory system and therefore dependent on other systemic factors like hypertension. Although most of the work performed refers to the mm-to-cm scale in space and to the second scale in time, the challenge has been set in integrating horizontally most of the patient-specific image information with domain knowledge on the physics of blood flow and of its interaction with medical devices. Future work should include extension of the vascular characterization to the whole circle of Willis (Piccinelli et al. 2009), the use of in vivo flow boundary conditions from non-invasive imaging modalities such as PC-MR (Funamoto et al. 2009), the inclusion of descriptors able to represent the peri-aneurysmal environment (San Millán Ruiz et al. 2006), and the fusion of multiple and complementary imaging modalities providing information on vascular structure, flow and its supporting environment. The integration of the structural and functional information across imaging modalities as well as the representation of fields resulting from the 
simulation outputs will be facilitated through the use of mark-up languages like FieldML (Christie et al. 2009). At the cellular scales, cellular models, like those provided by CellML (Lloyd et al. 2008; Garny et al. 2008), should be able to capture two different phenomena: blood coagulation and aneurysm growth (Watton et al. 2009b; Watton \& Ventikos 2009; Watton et al. 2009a). These, combined with the existing endovascular device models, should be able to provide further assessment of the patient aneurysmal rupture risk and evolution after treatment.

Finally, a number of software frameworks are emerging that will facilitate the creation of clinical application prototypes that streamline the complex processing chains derived from these workflows (Viceconti et al. 2007a,b; Larrabide et al. 2009). The presented pipeline is framed in the context of the VPH Initiative (Viceconti et al. 2008). On the one hand, all our current scientific and technical efforts aim at extending the present pipeline along the lines supporting the VPH vision, which aims at progressing towards a comprehensive and integrated view of human physiology that enables the delivery of sound diagnostic and prognostic decisions in health care management. On the other hand, the VPH initiative provides the framework that allows all these tools to converge, maximizing its impact on industry, as well as on the scientific and clinical communities. The existence of appropriate IT infrastructures will allow collecting data in a rational manner and establishing links with a broader scientific community. The European integrated project @neurIST is an example of this, where image-derived morphological, haemodynamical and structural descriptors are combined and cross-linked with clinical and genetic information underpinned and enabled through advanced IT infrastructures for data sharing and efficient reuse of computational resources (Arbona et al. 2006, 2007; Dunlop et al. 2008; Friedrich et al. 2008; Iavindrasana et al. 2008).

This work was partially supported by the @neurIST Integrated Project (co-financed by the European Commission through the contract no. IST-027703), the CDTI CENITCDTEAM grant funded by the Spanish Ministry of Science and Innovation (MICINNCDTI) and Philips Healthcare (Best, The Netherlands). The authors would also like to thank the support provided by ANSYS Inc. (Canonsburg, PA, USA).

\section{References}

Alastruey, J., Parker, K., Peiró, J., Byrd, S. \& Sherwin, S. 2007 Modelling the circle of Willis to assess the effects of anatomical variations and occlusions on cerebral flows. J. Biomech., 40(8), 1794-1805. (doi:10.1016/j.jbiomech.2006.07.008)

Antiga, L., Piccinelli, M., Botti, L., Ene-Iordache, B., Remuzzi, A. \& Steinman, D. 2008 An image-based modeling framework for patient-specific computational hemodynamics. Med. Biol. Eng. Comput., 46(11), 1097-1112. (doi:10.1007/ s11517-008-0420-1)

Arbona, A., Benkner, S., Engelbrecht, G., Fingberg, J., Hofmann, M., Kumpf, K., Lonsdale, G. \& Woehrer, A. 2007 A service-oriented grid infrastructure for biomedical data and compute services. IEEE Trans. Nanobioscience, 6(2), 136141.

Arbona, A., Benkner, S., Fingberg, J., Frangi, A. F., Hofmann, M., Hose, D. R., Lonsdale, G., Ruefenacht, D. \& Viceconti, M. 2006 Outlook for grid service tech- 
nologies within the @neurIST eHealth environment. Stud. Health Technol. Inform., 120, 401-404.

Balocco, S., Camara, O. \& Frangi, A. F. 2008 Towards regional elastography of intracranial aneurysms. In Med. Imag. Comp. Comp.-Assist. Interv. MICCAI, vol. 5242 of Lecture Notes on Computer Science, pp. 131-138. (doi:10.1007/ 978-3-540-85990-1)

Blanco, P. J., Feijóo, R. A. \& Urquiza, S. A. 2007 A unified variational approach for coupling 3D-1D models and its blood flow applications. Comput. Method Appl. M., 196(41-44), 4391-4410. (doi:10.1016/j.cma.2007.05.008)

Bogunović, H., Radaelli, A., De Craene, M., Delgado, D. \& Frangi, A. F. 2008 Image intensity standardization in 3D rotational angiography and its application to vascular segmentation. In SPIE Medical Imaging 2008: Image Processing (eds J. Reinhardt \& J. Pluim), vol. 6914, p. 691419. (doi:10.1117/12.770564)

Boussel, L., Rayz, V., McCulloch, C., Martin, A., Acevedo-Bolton, G., Lawton, M., Higashida, R., Smith, W. S., Young, W. L. et al. 2008 Aneurysm growth occurs at region of low wall shear stress: Patient-specific correlation of hemodynamics and growth in a longitudinal study. Stroke, 39(11), 2997-3002. (doi:10.1161/ STROKEAHA.108.521617)

Brisman, J., Song, J. \& Newell, D. 2006 Medical progress: cerebral aneurysms. New Engl. J. Med., 355(9), 928-939.

Brisman, J., Song, J., Niimi, Y. \& Berenstein, A. 2005 Treatment options for widenecked intracranial aneurysms using a self-expandable hydrophilic coil and a self-expandable stent combination. Am. J. Neuroradiol., 26(5), 1237-1240.

Calamante, F., Yim, P. \& Cebral, J. R. 2003 Estimation of bolus dispersion effects in perfusion MRI using image-based computational fluid dynamics. Neuroimage, 19(2), 341-353. (doi:10.1016/S1053-8119(03)00090-9)

Castro, M. A., Putman, C. M. \& Cebral, J. R. 2006 Patient-specific computational modeling of cerebral aneurysms with multiple avenues of flow from 3D rotational angiography images. Acad. Radiol., 13(7), 811-821. (doi:10.1016/j.acra.2006.03. 011)

Cebral, J., Castro, M., Satoh, T. \& Burgess, J. 2004 Evaluation of image-based CFD models of cerebral aneurysm using MRI. In ISMRM Flow Motion Workshop, pp. 11-13. Zurich, Switzerland.

Cebral, J. R., Castro, M. A., Appanaboyina, S., Putman, C. M., Millan, D. \& Frangi, A. F. 2005 Efficient pipeline for image-based patient-specific analysis of cerebral aneurysm hemodynamics: technique and sensitivity. IEEE Trans. Med. Imag., 24(4), 457-467. (doi:10.1109/TMI.2005.844159)

Cebral, J. R. \& Löhner, R. 2005 Efficient simulation of blood flow past complex endovascular devices using an adaptive embedding technique. IEEE Trans. Med. Imag., 24(4), 468-476. (doi:10.1109/TMI.2005.844172) 
Cebral, J. R., Pergolizzi, R. \& Putman, C. M. 2007 Computational fluid dynamics modeling of intracranial aneurysms: qualitatively comparison with cerebral angiography. Acad. Radiol., 14(7), 804-813. (doi:10.1016/j.acra.2007.03.008)

Chang, H.-H., Duckwiler, G. R., Valentino, D. J. \& Chu, W. C. 2009 Computerassisted extraction of intracranial aneurysms on 3D rotational angiograms for computational fluid dynamics modeling. Med. Phys., 36(12), 5612-5621. (doi: $10.1118 / 1.3260841)$

Christie, G. R., Nielsen, P. M., Blackett, S. A., Bradley, C. P. \& Hunter, P. J. 2009 FieldML: concepts and implementation. Proc. R. Soc. A, 367(1895), 1869-1884. (doi:10.1098/rsta.2009.0025)

De Craene, M., Camara, O., Bijnens, B. H. \& Frangi, A. F. 2009 Non-stationary diffeomorphic registration: application to endovascular treatment monitoring. In Medical imaging 2009: Image processing (eds J. P. W. Pluim \& B. M. Dawant), vol. 7259, p. 72591F. SPIE.

De Craene, M., Pozo, J. M., Villa-Uriol, M. C., Vivas, E., Sola, T., Guimaraens, L., Blasco, J., Macho, J. \& Frangi, A. F. 2008 Coil compaction and aneurysm growth: image-based quantification using non-rigid registration. In Medical imaging 2008: Computer-aided diagnosis (eds M. Giger \& N. Karssemeijer), vol. 6915, p. 69151R. SPIE. (doi:10.1117/12.770637)

Delingette, H. 1999 General object reconstruction based on simplex meshes. Int. J. Comp. Vis., 32(2), 111-146.

Dempere-Marco, L., Oubel, E., Castro, M. A., Putman, C. M., Millan, R. D. \& Frangi, A. F. 2006 CFD analysis incorporating the influence of wall motion: application to intracranial aneurysms. In Med. Imag. Comp. Comp.-Assist. Interv. MICCAI, vol. 4191 of Lecture Notes on Computer Science, pp. 438-445. (doi: $\left.10.1007 / 11866763 \backslash \_54\right)$

Dunlop, R., Arbona, A., Rajasekaran, H., Lo Iacono, L., Fingberg, J., Summers, P., Benkner, S., Engelbrecht, G., Chiarini, A. et al. 2008 @neurIST - Chronic disease management through integration of heterogeneous data and computerinterpretable guideline services. Stud. Health Technol. Inform., 138, 173-177.

Fenner, J., Brook, B., Clapworthy, G., Coveney, P., Feipel, V., Gregersen, H., Hose, D., Kohl, P., Lawford, P. et al. 2008 The EuroPhysiome, STEP and a roadmap for the virtual physiological human. Proc. R. Soc. A, 366(1878), 2979-2999. (doi:10.1098/rsta.2008.0089)

Flore, E., Larrabide, I., Petrini, L., Pennati, G. \& Frangi, A. F. 2009 Stent deployment in aneurysmatic cerebral vessels: Assessment and quantification of the differences between Fast Virtual Stenting and Finite Element Analysis. In CI2BM09 - MICCAI Workshop on Cardiovascular Interventional Imaging and Biophysical Modelling, vol. 5242, pp. 790-797.

Ford, M., Alperin, N., Lee, S., Holdsworth, D. \& Steinman, D. $2005 a$ Characterization of volumetric flow rate waveforms in the normal internal carotid and vertebral arteries. Physiol. Meas., 26(4), 477-488. (doi:10.1088/0967-3334/26/4/013) 
Ford, M., Stuhne, G., Nikolov, H., Habets, D., Lownie, S., Holdsworth, D. \& Steinman, D. $2005 b$ Virtual angiography for visualization and validation of computational models of aneurysm hemodynamics. IEEE Trans. Med. Imag., 24(12), 1586-1592. (doi:10.1109/TMI.2005.859204)

Ford, M. D., Nikolov, H. N., Milner, J. S., Lownie, S. P., DeMont, E. M., Kalata, W., Loth, F., Holdsworth, D. W. \& Steinman, D. A. 2008 PIV-measured versus CFD-predicted flow dynamics in anatomically realistic cerebral aneurysm models. J. Biomech. Eng., 130(2), 021015. (doi:10.1115/1.2900724)

Friedrich, C. M., Dach, H., Gattermayer, T., Engelbrecht, G., Benkner, S. \& Hofmann-Apitius, M. 2008 @neuLink: a service-oriented application for biomedical knowledge discovery. Stud. Health Technol. Inform., 138, 165-172.

Funamoto, K., Suzuki, Y., Hayase, T., Kosugi, T. \& Isoda, H. 2009 Numerical evaluation of MR-measurement-integrated simulation of unsteady hemodynamics in a cerebral aneurysm. In Int. Conf. Biomed. Eng., vol. 23 of IFMBE Proceedings, pp. 2188-2191. (doi:10.1007/978-3-540-92841-6_547)

Ganguly, A., Rudin, S., Bednarek, D., Hoffmann, K. \& Kyprianou, I. 2003 Microangiography for neuro-vascular imaging. I. Experimental evaluation and feasibility. Med. Phys., 30(11), 3018-3028. (doi:10.1118/1.1617549)

Garny, A., Nickerson, D. P., Cooper, J., Weber dos Santos, R., Miller, A. K., McKeever, S., Nielsen, P. M. \& Hunter, P. J. 2008 CellML and associated tools and techniques. Proc. R. Soc. A, 366(1878), 3017-3043. (doi:10.1098/rsta.2008.0094)

Geers, A., Larrabide, I., Radaelli, A. G., Bogunović, H., Gratama van Andel, H. A. F., Majoie, C. B. \& Frangi, A. F. 2009 Reproducibility of image-based computational hemodynamics in intracranial aneurysms: comparison of CTA and 3DRA. In IEEE Int. Symp. Biomed. Imag., pp. 610-613. Boston, MA, USA.

Guglielmi, G., Viñuela, F., Dion, J. \& Duckwiler, G. 1991 Electrothrombosis of saccular aneurysms via endovascular approach. Part 2: Preliminary clinical experience. J. Neurosurg., 75(1), 8-14. (doi:10.3171/jns.1991.75.1.0008)

Hayakawa, M., Katada, K., Anno, H., Imizu, S., Hayashi, J., Irie, K., Negoro, M., Kato, Y., Kanno, T. et al. 2005 CT angiography with electrocardiographically gated reconstruction for visualizing pulsation of intracranial aneurysms: identification of aneurysmal protuberance presumably associated with wall thinning. Am. J. Neuroradiol., 26, 1366-1369.

Hernandez, M. \& Frangi, A. F. 2007 Non-parametric geodesic active regions: Method and evaluation for cerebral aneurysms segmentation in 3DRA and CTA. Med. Image Anal., 11(3), 224-241. (doi:10.1016/j.media.2007.01.002)

Hoi, Y., Woodward, S., Kim, M., Taulbee, D. \& Meng, H. 2006 Validation of CFD simulations of cerebral aneurysms with implication of geometric variations. $J$. Biomech. Eng., 128(6), 844-851. (doi:10.1115/1.2354209)

Hunter, P. \& Borg, T. 2003 Integration from proteins to organs: The Physiome Project. Nature, 4(3), 237-243. (doi:10.1038/nrm1017) 
Hunter, P., Robbins, P. \& Noble, D. 2002 The IUPS Human Physiome Project. Eur. J. Physiol., 445(1), 1-9. (doi:10.1007/s00424-002-0890-1)

Iavindrasana, J., Lo Iacono, L., Müller, H., Periz, I., Summers, P., Wright, J., Friedrich, C., Dach, H., Gattermayer, T. et al. 2008 The @neurIST project. Stud. Health Technol. Inform., 138, 161-164.

Jou, L.-D., Quick, C. M., Young, W. L., Lawton, M. T., Higashida, R. T., Martin, A., \& Saloner, D. 2003 Computational approach to quantifying hemodynamic forces in giant cerebral aneurysms. Am. J. Neuroradiol., 24(9), 1804-1810.

Juvela, S. 2003 Prehemorrhage risk factors for fatal intracranial aneurysm rupture. Stroke, 34(8), 1852-1858. (doi:10.1161/01.STR.0000080380.56799.DD)

Kakalis, N. M., Mitsos, A. P., Byrne, J. V. \& Ventikos, Y. 2008 The haemodynamics of endovascular aneurysm treatment: a computational modelling approach for estimating the influence of multiple coil deployment. IEEE Trans. Med. Imag., 27(6), 814-824. (doi:10.1109/TMI.2008.915549)

Kayembe, K., Sasahara, M. \& Hazama, F. 1984 Cerebral aneurysms and variations in the circle of Willis. Stroke, 15(5), 846-850.

Kim, M., Larrabide, I., Villa-Uriol, M. C. \& Frangi, A. F. 2009 Hemodynamic alterations of a patient-specific intracranial aneurysm induced by virtual deployment of stents in various axial orientation. In IEEE Int. Symp. Biomed. Imag. (doi:10.1109/ISBI.2009.5193280)

Kim, M., Taulbee, D., Tremmel, M. \& Meng, H. 2008 Comparison of two stents in modifying cerebral aneurysm hemodynamics. In Ann. Biomed. Eng., vol. 36, pp. 726-741. (doi:10.1007/s10439-008-9449-4)

Krissian, K., Bogunović, H., Pozo, J. M., Villa-Uriol, M. C. \& Frangi, A. F. 2008 Minimally interactive knowledge-based coronary tracking in CTA using a minimal cost path. The Midas Journal. In: 2008 MICCAI Workshop Grand Challenge Coronary Artery Tracking.

Kroon, M. \& Holzapfel, G. A. 2008 Estimation of the distributions of anisotropic, elastic properties and wall stresses of saccular cerebral aneurysms by inverse analysis. Proc. R. Soc. A, 464(2092), 807-825. (doi:10.1098/rspa.2007.0332)

Larrabide, I., Omedas, P., Martelli, Y., Planes, X., Nieber, M., Moya, J. A., Butakoff, C., Sebastián, R., Camara, O. et al. 2009 GIMIAS: An open source framework for efficient development of research tools and clinical prototypes. In Functional Imaging and Modeling of the Heart, vol. 5528/2009 of Lecture Notes in Computer Science, pp. 417-426. (doi:10.1007/978-3-642-01932-6)

Larrabide, I., Radaelli, A. G. \& Frangi, A. F. 2008 Fast virtual stenting with deformable meshes: Application to intracranial aneurysms. In Med. Imag. Comp. Comp.-Assist. Interv. MICCAI, vol. 5242 of Lecture Notes in Computer Science, pp. 790-797. (doi:10.1007/978-3-540-85990-1) 
Liang-Der, J., Saloner, D. \& Higashida, R. 2004 Determining intra-aneurysmal flow for coiled cerebral aneurysm with Digital Fluoroscopy. Biomed. Eng. Appl. Basis Comm., 16(2), 43-48. (doi:10.4015/S1016237204000086)

Liou, T. M. \& Li, Y. C. 2008 Effects of stent porosity on hemodynamics in a sidewall aneurysm model. J. Biomech. Eng., 41(6), 1174-11 837. (doi:10.1016/j.jbiomech. 2008.01.025)

Lloyd, C. M., Lawson, J. L., Hunter, P. J. \& Nielsen, P. F. 2008 The CellML model repository. Bioinformatics, 24(18), 2122-2123. (doi:10.1093/bioinformatics/ btn390)

Lo, C. \& Don, H. 1989 3-D moments forms: Their construction and application to object identification and positioning. IEEE Trans. Pattern Anal. Machine Intell., 11(10), 1053-1064. (doi:10.1109/34.42836)

Lylyk, P., Ferrario, A., Pasbon, B., Miranda, C. \& Doroszuk, G. 2005 Buenos Aires experience with the Neuroform self-expanding stent for the treatment of intracranial aneurysms. J. Neurosurg., 102(2), 235-241.

Ma, B., Harbaugh, R. E. \& Raghavan, M. L. 2004 Three-dimensional geometrical characterization of cerebral aneurysms. Ann. Biomed. Eng., 32(2), 264-273.

Meng, H., Wang, Z., Hoi, Y., Gao, L., Metaxa, E., Swartz, D. \& Kolega, J. 2007 Complex hemodynamics at the apex of an arterial bifurcation induces vascular remodeling resembling cerebral aneurysm initiation. Stroke, 38, 1924-1931. (doi: 10.1161/STROKEAHA.106.481234)

Millan, R., Dempere-Marco, L., Pozo, J. M., Cebral, J. R. \& Frangi, A. F. 2007 Morphological characterization of intracranial aneurysms using 3-D moment invariants. IEEE Trans. Med. Imag., 26(9), 1270-1282. (doi:10.1109/TMI.2007. 901008)

Morales, H., Kim, M., Villa-Uriol, M. C., Vivas, E. \& Frangi, A. F. 2009 Influence of coil packing rate and configuration on intracranial aneurysm hemodynamics. In 11th International Congress of the IUPESM, Medical Physics and Biomedical Engineering, World Congress 2009.

Narracott, A., Smith, S., Lawford, P., Liu, H., Himeno, R., Wilkinson, I., Griffiths, P. \& Hose, R. 2005 Development and validation of models for the investigation of blood clotting in idealized stenoses and cerebral aneurysms. J. Artif. Organs, 8, 56-62. (doi:10.1007/s10047-004-0274-8)

Novotni, M. \& Klein, R. 2004 Shape retrieval using 3D Zernike descriptors. Computed-Aided Design, 36, 1047-1062.

Olufsen, M. S., Nadim, A. \& Lipsitz, L. A. 2002 Dynamics of cerebral blood flow regulation explained using a lumped parameter model. Am. J. Physiol., Reg. Int. Comp. Physiol., 282, R611-R622. 
Oubel, E., De Craene, M., Putman, C. M., Cebral, J. R. \& Frangi, A. F. 2007 Analysis of intracranial aneurysm wall motion and its effects on hemodynamic patterns. In SPIE Medical Imaging: Physics of Medical Imaging Image Reconstruction (eds A. Manduca \& X. P. Hu), vol. 6511 of Lecture Notes on Computer Science, p. 65112A. (doi:10.1117/12.708937)

Piccinelli, M., Veneziani, A., Steinman, D., Remuzzi, A. \& Antiga, L. 2009 A framework for geometric analysis of vascular structures: application to cerebral aneurysms. IEEE Trans. Med. Imag., 28(8), 1141-1155. (doi:10.1109/TMI.2009. 2021652)

Piotin, M., Mandai, S., Murphy, K. J., Sugiu, K., Gailloud, P., Martin, J. B. \& Rüfenacht, D. A. 2000 Dense packing of cerebral aneurysms: an in vitro study with detachable platinum coils. Am. J. Neuroradiol., 21, 757-760.

Radaelli, A., Augsburger, L., Cebral, J., Ohta, M., Rüfenacht, D., Balossino, R., Benndorf, G., Hose, D., Marzo, A. et al. 2008 Reproducibility of haemodynamical simulations in a subject-specific stented aneurysm model A report on the Virtual Intracranial Stenting Challenge 2007. J. Biomech., 41(10), 2069-2081. (doi: 10.1016/j.jbiomech.2008.04.035)

Radaelli, A. G., Sola Martínez, T., Vivas Díaz, E., Mellado, X., Castro, M. A., Putman, C. M., Guimaraens, L., Cebral, J. R. \& Frangi, A. F. 2007 Combined clinical and computational information in complex cerebral aneurysms: application to mirror cerebral aneurysms. In SPIE Medical Imaging: Physiology, Function, and Structure from Medical Images (eds A. Manduca \& X. P. Hu), vol. 6511 of Lecture Notes on Computer Science, p. 65111F. (doi:10.1117/12.708955)

Raghavan, M. L., Ma, B. \& Harbaugh, R. E. 2005 Quantified aneurysm shape and aneurysm rupture. J. Neurosurg., 102(2), 355-362.

Raymond, J., Guilbert, F., Weill, A., Georganos, S. A., Juravsky, L., Lambert, A., Lamoureux, J., Chagnon, M. \& Roy, D. 2003 Long-term angiographic recurrences after selective endovascular treatment of aneurysms with detachable coils. Stroke, 34, 1398-1403. (doi:10.1161/01.STR.0000073841.88563.E9)

Rohde, S., Lahmann, K., Beck, J., Nafe, R., Yan, B., Raabe, A. \& Berkefeld, J. 2005 Fourier analysis of intracranial aneurysms: towards an objective and quantitative evaluation of the shape of aneurysms. Neuroradiol., 47, 121-126.

Rudin, S., Bednarek, D. \& Hoffmann, K. 2008 Endovascular image-guided interventions (EIGIs). Med. Phys., 35(1), 301-309.

San Millán Ruiz, D., Yilmaz, H., Dehashti, A., Alimenti, A., de Tribolet, N. \& Rüfenacht, D. 2006 The perianeurysmal environment: influence on saccular aneurysm shape and rupture. Am. J. Neuroradiol., 27(3), 504-512.

Satoh, T., Onoda, K. \& Tsuchimoto, S. 2004 Visualization of intraaneurysmal flow patterns with transluminal flow images of 3D MR angiograms in conjunction with aneurysmal configurations. Am. J. Neuroradiol., 24(7), 1436-1445. 
Schaap, M., Metz, C., van Walsum, T., van der Giessen, A., Weustink, A., Mollet, N., Bauer, C., Bogunović, H., Castro, C. et al. 2009 Standardized evaluation methodology and reference database for evaluating coronary artery centerline extraction algorithms. Med. Image Anal., 13, 701-714. (doi:10.1016/j.media. 2009.06.003)

Singh, P., Marzo, A., Coley, S., Berti, G., Bijlenga, P., Lawford, P., Villa-Uriol, M. C., Rüfenacht, D., McCormack, K. et al. 2009 The role of computational fluid dynamics in the management of unruptured intracranial aneurysms: a clinicians view. Comput. Intell. Neurosci., 2009(760364), 1-12. (doi:10.1155/2009/760364)

Sluzewski, M., van Rooij, W. J., Slob, M. J., Bescós, J. O., Slump, C. H. \& Wijnalda, D. 2004 Relation between aneurysm volume, packing, and compaction in 145 cerebral aneurysms treated with coils. Radiology, 231, 653-658. (doi:10.1148/ radiol.2313030460)

Steinman, D., Milner, J., Norley, C., Lownie, S. \& Holdsworth, D. 2003 Image-based computational simulation of flow dynamics in a giant intracranial aneurysm. Am. J. Neuroradiol., 24, 559-566.

Stuhne, G. R. \& Steinman, D. A. 2004 Finite-element modeling of the hemodynamics of stented aneurysms. J. Biomech. Eng., 126(3), 382-387.

Taylor, C. \& Humphrey, J. 2009 Open problems in computational vascular biomechanics: Hemodynamics and arterial wall mechanics. Comput. Methods Appl. Mech. Engrg., 198, 3514-3523. (doi:10.1016/j.cma.2009.02.004)

Ujiie, H., Tachibana, H., Hiramatsu, O., Hazel, A. L., Matsumoto, T., Ogasawara, Y., Nakajima, H., Hori, T., Takakura, K. et al. 1999 Effects of size and shape (aspect ratio) on the hemodynamics of saccular aneurysms: A possible index for surgical treatment of intracranial aneurysms. Neurosurgery, 45(1), 119-130.

Vanninen, R., Manninen, H. \& Ronkainen, A. 2003 Broad-based intracranial aneurysms: Thrombosis induced by stent placement. Am. J. Neuroradiol., 24, 263-266.

Viceconti, M., Clapworthy, G. \& Van Sint Jan, S. 2008 The Virtual Physiological Human - a European initiative for in silico human modelling -. J. Physiol. Sci., 58(7), 441-447. (doi:10.2170/physiolsci.RP009908)

Viceconti, M., Taddei, F., Montanari, L., Testi, D., Leardini, A., Clapworthy, G. \& Van Sint Jan, S. 2007 a Multimod Data Manager: a tool for data fusion. Comput. Methods Programs Biomed., 87(2), 148-159. (doi:10.1016/j.cmpb.2007.05.002)

Viceconti, M., Zannoni, C., Testi, D., Petrone, M., Perticoni, S., Quadrani, P., Taddei, F., Imboden, S. \& Clapworthy, G. 2007b The Multimod Application Framework: a rapid application development tool for computer aided medicine. Comput. Methods Programs Biomed., 85(2), 138-151. (doi:10.1016/j.cmpb.2006. 09.010)

Watton, P. \& Ventikos, Y. 2009 Modelling evolution of saccular cerebral aneurysms. J. Strain Anal. Eng., 44(5), 375-389. (doi:10.1243/03093247JSA492) 
Watton, P. N., Raberger, N. B., Holzapfel, G. A. \& Ventikos, Y. $2009 a$ Coupling the hemodynamic environment to the evolution of cerebral aneurysms: Computational framework and numerical examples. J. Biomech. Eng., 131(10), 101003. (doi:10.1115/1.3192141)

Watton, P. N., Ventikos, Y. \& Holzapfel, G. A. $2009 b$ Modelling the growth and stabilization of cerebral aneurysms. Math. Med. Biol., 26(2), 133-164. (doi: 10.1093/imammb/dqp001)

Zhang, C., De Craene, M., Villa-Uriol, M. C., Pozo, J. M., Bijnens, B. H. \& Frangi, A. F. 2009a Estimating continuous 4D wall motion of cerebral aneurysms from 3D rotational angiography. In Med. Imag. Comp. Comp.-Assist. Interv. MICCAI (ed. G.-Z. Y. et al.), vol. 5761 of Lecture Notes on Computer Science, pp. 140-147. (doi:10.1007/978-3-642-04268-3)

Zhang, C., Villa-Uriol, M. C., De Craene, M., Pozo, J. M. \& Frangi, A. F. $2009 b$ Morphodynamic analysis of cerebral aneurysm pulsation from time-resolved rotational angiography. IEEE Trans. Med. Imag., 28(7), 1105-1116. (doi: 10.1109/TMI.2009.2012405) 